\title{
Incidental Angiosarcoma of the Pancreas: A Case Report of a Rare, Asymptomatic Tumor
}

\author{
Patrick J. Worth, ${ }^{1}$ Michael Turner, ${ }^{1}$ and Chet W. Hammill ${ }^{2, *}$
}

\begin{abstract}
Background: Angiosarcoma of the pancreas is a very rare neoplasm accounting for $0.1 \%$ of pancreatic malignancies. This tumor is biologically very aggressive and frequently diagnosed at an unresectable stage.

Case Presentation: Herein, we report a case of an incidentally discovered angiosarcoma that was removed with a robotic distal pancreatectomy and discuss the current literature on this rare disease.

Conclusion: This is the fifth reported case of primary angiosarcoma of the pancreas and the first case to be identified incidentally. Unlike the other cases where survival was limited due to advanced disease, the patient presented here underwent surgical resection and remains disease free after 1 year of follow-up.
\end{abstract}

Keywords: angiosarcoma of the pancreas; distal pancreatectomy; robotic pancreatic surgery

\section{Introduction}

Angiosarcoma is classically thought of as a neoplasm of the head and neck, occurring most frequently in the elderly. ${ }^{1}$ Primary sarcomata of the pancreas are very rare, accounting for $0.1 \%$ of pancreatic malignancies, ${ }^{2}$ and angiosarcomas account for less than $1 \%$ of all sarcomas. ${ }^{3}$ Primary angiosarcoma of the pancreas was first reported in Romania by Bancu et al. in 1971, ${ }^{4}$ and since then only four cases have been reported. ${ }^{3,5-7}$ Angiosarcomas are fast growing tumors and, when involving the gastrointestinal tract, have nonspecific symptoms such as gastrointestinal bleeding, anemia, weight loss, and abdominal pain. ${ }^{1,3}$ As a result, they are often diagnosed at an advanced stage and their prognosis is poor, with a median survival of less than 12 months. ${ }^{1-3}$ The only known curative treatment is surgical resection, which is limited to localized disease. ${ }^{2,3}$

\section{Case Report}

We report the case of a 78-year-old female with an incidentally identified angiosarcoma of the pancreas. The pa- tient's medical history was significant for hypertension and stage III breast cancer. Her breast cancer was diagnosed in 1994 and she was treated with lumpectomy, axillary lymph node dissection, and $5000 \mathrm{cGy}$ whole-breast radiation with a $1000 \mathrm{cGy}$ boost for 44 days, with no evidence of recurrence. Her surgical history was also significant for an appendectomy and salpingo-oophorectomy for a serous cyst of the left ovary. In November of 2015, she presented to her primary care physician with swollen inguinal lymph nodes, and an abdominal ultrasonography was performed. The ultrasonography incidentally identified a $1 \mathrm{~cm}$ cystic lesion in the head of the pancreas, prompting a pancreas-protocol computed tomography (CT) scan. In addition to a $1 \mathrm{~cm}$ cystic lesion in the head of the pancreas, the CT identified a $3.1 \times 2.4 \mathrm{~cm}$ lesion on the anterior surface of the pancreatic tail. The lesion was enhancing in both the arterial and venous phases of the CT and abutted the splenic flexure of the colon and the posterior wall of the stomach (Fig. 1). Of note, the lesion was not visible on a CT with contrast from 2009. An MRI was performed, which further

${ }^{1}$ Department of Surgery, Oregon Health and Science University, Portland, Oregon.

${ }^{2}$ Department of Surgery, Washington University in Saint Louis School of Medicine, Saint Louis, Missouri.

*Address correspondence to: Chet W. Hammill, MD, MCR, Department of Surgery, Washington University in Saint Louis School of Medicine, 660 S. Euclid Avenue, Campus Box 8109, Saint Louis, MO 63110, E-mail: hammill@wustl.edu

() Patrick J. Worth et al. 2017; Published by Mary Ann Liebert, Inc. This is an Open Access article distributed under the terms of the Creative Commons Attribution License, which permits unrestricted use, distribution, and reproduction in any medium, provided the original work is properly cited. Mary Ann Liebert, Inc. offers reprint services for those who want to order professionally produced copies of articles published under the Creative Commons Attribution (CC BY) license. To obtain a price quote, email Reprints@liebertpub.com. Please include the article's title or DOI, quantity, and delivery destination in your email. 

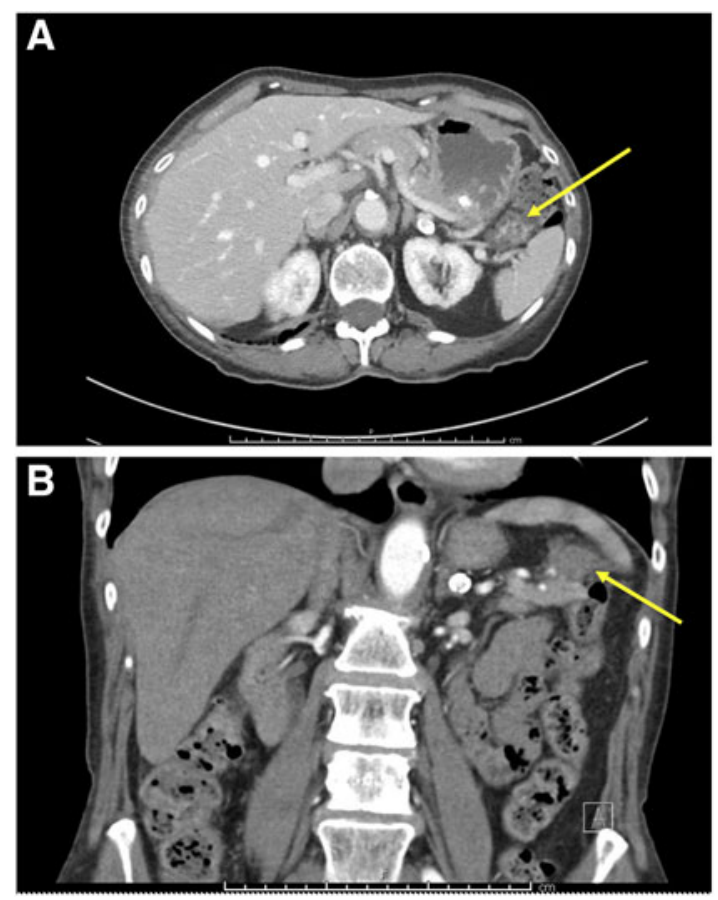

FIG. 1. (A) Representative venous phase (axial) contrasted CT scan showing the tail of pancreas lesion (arrow) and (B) representative arterial phase, coronal reconstruction. $\mathrm{CT}$, computed tomography.

delineated a nonspecific irregular mass arising from the anterior surface of the tail of the pancreas, with features consistent with a mucinous cystic neoplasm or a variant of ductal adenocarcinoma. Endoscopic ultrasonography again identified the $1 \mathrm{~cm}$ cystic lesion in the head of the pancreas, which was consistent with serous cystadenoma on fine-needle aspiration. Examination of the pancreatic tail revealed a cystic-appearing lesion measuring $7 \times 5 \mathrm{~mm}$ with well-defined endoscopic borders and surrounding hyperechoic regions. Fine-needle aspirate of the tail lesion was suspicious for adenocarcinoma.

The patient's preoperative history was notably absent of weight loss, back pain, jaundice, or new-onset diabetes. She was a former smoker, drinks socially, and has no significant family history. Informed consent was provided and she was taken to the operating room for exploration and resection of the distal pancreas lesion. Intraoperatively, a lesion in segment II of the liver was identified and a core-needle biopsy was obtained; pathology analysis was negative for malignancy; the lesion corresponded to a $9 \times 6 \mathrm{~mm}$ low-attenuation lesion seen on a CT scan done several days before surgery. The pancreatic tail mass appeared hypervascular with pale tan parenchyma; it was firm but easily dissected away from the stomach and colon. The lesion involved the tail of the pancreas and after dissection was noted to be in close association with the splenic vasculature. Based on this, a roboticassisted distal pancreatectomy and splenectomy were completed. After completing the resection, the distal pancreas and mass were separated from the spleen intracorporeally, placed in a specimen bag, and removed en bloc through a small extension of a $15-\mathrm{mm}$ trocar site. The spleen was morcellated in a second specimen bag and removed. The patient's postoperative course was uncomplicated and she was discharged to home on postoperative day 4 .

Pathology analysis demonstrated a $2.4 \times 1.6 \times 1.6 \mathrm{~cm}$ "vascular neoplasm with cystic change" (Fig. 2A, B); all margins were negative for tumor. Immunohistochemical studies performed on representative sections of the
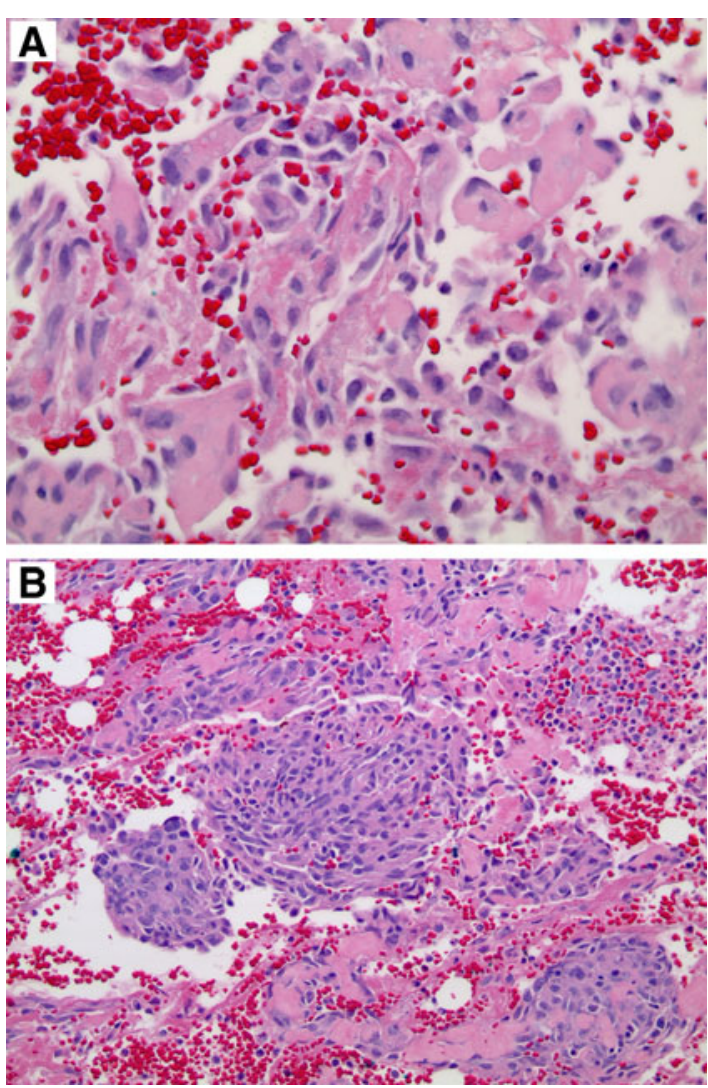

FIG. 2. Representative $H \& E$ sections from the tumor. (A) $20 \times$ representative section showing endothelial morphology and pleomorphic nuclei. (B) $10 \times$ imaging. 

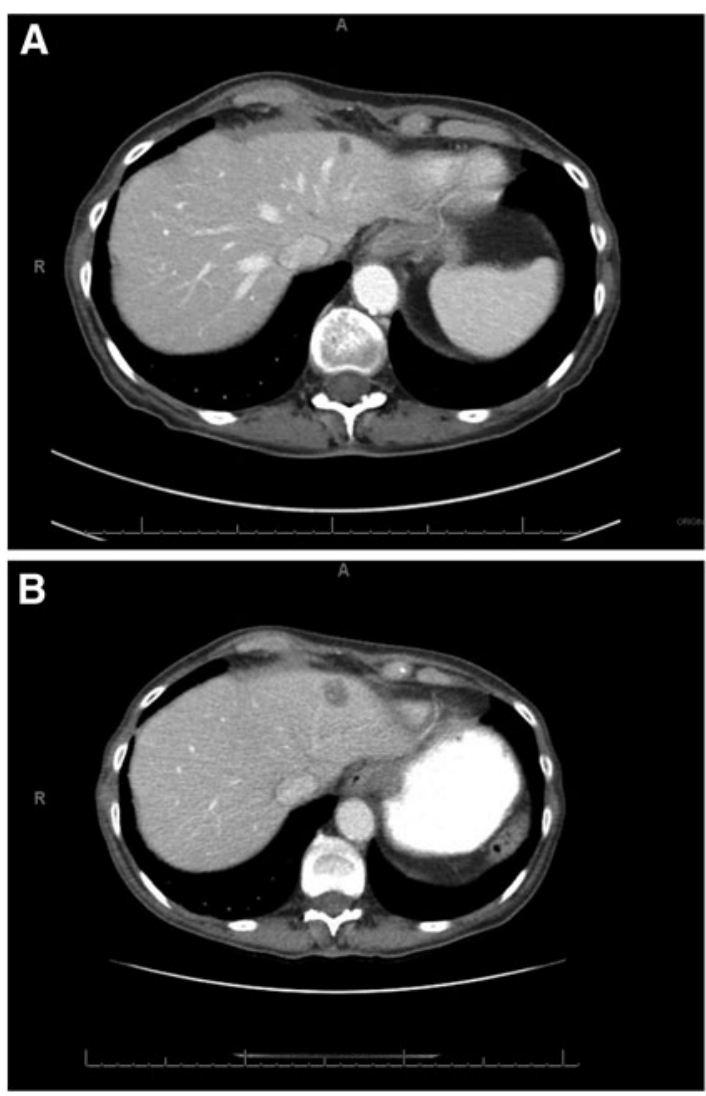

FIG. 3. Interval change in venous-contrasted phase of $\mathrm{CT}$ abdomen showing growth in the segment II liver lesion. (A) initial scan, (B) 4 months postoperation.

cystic neoplasm demonstrate a moderate increase in Ki-67 proliferation and positive staining for endothelial markers CD31 and CD34. Immunological staining for epithelial markers cytokeratin pool, CK5/6, as well as S-100, melan-A, chromogranin, and synaptophysin was negative. Outside review of the pathology analysis found that the lesion was most consistent with an angiosarcoma arising from a vascular malformation of the pancreas.

Based on presentation at our multidisciplinary tumor board, a recommendation was made for close surveillance for recurrence and no adjuvant therapy. Of note, a follow-up CT scan of the abdomen and pelvis with contrast performed 4 months after surgery showed interval enlargement of the segment II liver lesion that was biopsied during surgery (Fig. 3). She was taken back to the operating room where she underwent a robot-assisted left lateral segmentectomy. She tolerated surgery without problem and was discharged home on postoperative day 4. Final pathology analysis of the liver lesion demonstrated a $2.4 \mathrm{~cm}$ benign cavernous hemangioma with organizing thrombus and scattered vascular malformations, lacking features of the previous malignant angiosarcoma. At 1 year follow-up from her pancreatic resection, she continued to do well with no evidence of recurrence.

\section{Discussion}

Angiosarcoma is a rare vascular neoplasm usually arising in the soft tissue or as a secondary malignancy in irradiated fields (head, neck, and breast). Primary angiosarcoma of the abdominal viscera is exceedingly rare and is most commonly seen in the liver or spleen. A 2010 review article that pooled data from the published literature identified 534 cases of angiosarcoma. Head and neck primaries were most common, accounting for $27 \%$ of cases, followed by breast primaries (20\%). Six percent were angiosarcomas of the liver and $2.6 \%$ originated in the spleen. ${ }^{7}$ The case we present is the fifth reported in the literature of angiosarcoma arising in the pancreas (Table 1). In addition, to our knowledge, it is the only case that has been diagnosed

Table 1. Reported Cases of Primary Angiosarcoma of the Pancreas

\begin{tabular}{|c|c|c|c|c|}
\hline Case & $\begin{array}{l}\text { Age/ } \\
\text { gender }\end{array}$ & Presentation & Treatment & Outcome \\
\hline 1. Csiskó et al. ${ }^{2}$ & $58 \mathrm{M}$ & $\begin{array}{l}\text { Severe acute pancreatitis, necrotizing } \\
\text { infection requiring laparotomy, } \\
\text { incidentally found mass }\end{array}$ & $\begin{array}{l}\text { Attempted tail-of-pancreas resection, } \\
\text { conservative management }\end{array}$ & $\begin{array}{l}\text { Death on postoperative day } \\
28 \text { secondary to } \\
\text { hemorrhagic shock }\end{array}$ \\
\hline 2. Seth et al. ${ }^{3}$ & $83 F$ & $\begin{array}{l}\text { Recurrent Gl bleeding, hemobilia, } \\
\text { history of gallstone pancreatitis }\end{array}$ & $\begin{array}{l}\text { Pylorus preserving } \\
\text { pancreaticoduodenectomy }\end{array}$ & Death on postoperative day 15 \\
\hline 3. Maeyashiki et al. ${ }^{5}$ & $72 \mathrm{M}$ & Dizziness, melena, severe anemia & $\begin{array}{l}\text { Attempted resection, aborted } \\
\text { because of multifocal } \\
\text { disseminated disease }\end{array}$ & $\begin{array}{l}\text { Death on hospital day } 103 \\
\text { because of hemorrhagic } \\
\text { shock }\end{array}$ \\
\hline 4. Meeks et al. ${ }^{6}$ & $65 \mathrm{M}$ & Severe acute abdominal pain & Pancreaticoduodenectomy & Death on postoperative day 6 \\
\hline 5. Current case & $78 \mathrm{~F}$ & $\begin{array}{l}\text { Incidentally found on abdominal } \\
\text { imaging for inguinal lymphadenopathy }\end{array}$ & $\begin{array}{l}\text { Robotic distal } \\
\text { pancreaticosplenectomy }\end{array}$ & Disease free after 1 year \\
\hline
\end{tabular}


incidentally. In most case reports, patient symptoms vary based on location of the tumor and can be associated with pain, jaundice, anorexia, and gastrointestinal or intraabdominal hemorrhage. ${ }^{2}$

Angiosarcomas stain positively for vascular (e.g., Factor VIII-related antigen, BNH9, CD34, and CD31) and negatively for epithelial markers (e.g., epithelial membrane antigen), and frequently have a subtle transition from a benign hemangiomatous neoplasm to the epithelioid or dissecting morphology seen in malignant disease. ${ }^{8}$ The finding of an associated vascular malformation may be of clinical interest on diagnostic imaging; however, it is unlikely to ultimately alter management. The mainstay of treatment of angiosarcoma follows guidelines for treatment of other soft tissue malignancies: complete resection and close monitoring for recurrence. In a large retrospective series published by Memorial SloanKettering, a survival benefit was demonstrated in surgical resection patients with negative microscopic margins compared with patients with positive microscopic margins. ${ }^{9}$ In this series, there was no benefit to adjuvant radiation for microscopically positive margins. The literature on the treatment of recurrent soft tissue malignancies may be extrapolated to the treatment of angiosarcoma in the setting of recurrence, with a limited but potential benefit derived from metastectomy. ${ }^{10,11}$

Although most angiosarcomas arise spontaneously, there are several known risk factors including radiation, chronic lymphedema, certain familial syndromes (neurofibromatosis, Maffucci syndrome, and KlippelTrenaunay syndrome), and several chemical carcinogens. ${ }^{7}$ Most notable of the chemical carcinogens are thorium dioxide and vinyl chloride, which are associated with hepatic angiosarcomas. Our patient did have a history of radiation a decade prior for breast-conserving treatment of her stage III ductal carcinoma. This raises the possibility of a "missed" primary angiosarcoma of the chest wall with a metastasis to the pancreas, which could be considered more likely than a primary angiosarcoma of the pancreas. However, a clinical breast examination, diagnostic mammogram performed postoperatively, and 1 year of follow-up to date have not produced any evidence for a primary angiosarcoma of the breast.

In summary, this is the fifth reported case of primary angiosarcoma of the pancreas and the first case to be identified incidentally. Unlike the other reported cases wherein survival was extremely limited because of advanced disease at presentation, the patient presented here underwent complete resection and has no evidence of recurrent disease after 1 year of follow-up.

\section{Author Disclosure Statement}

No competing financial interests exist.

\section{References}

1. Allison $\mathrm{K}$, et al. Angiosarcoma involving the gastrointestinal tract. A series of primary and metastatic cases. Am J Surg Pathol. 2004;28:298-307.

2. Csiskó $A$, et al. Primary angiosarcoma of the pancreas mimicking severe acute pancreatitis-case report. Pancreatology. 2015;15:84-87.

3. Seth A, Argani P, Campbell K. Angiosarcoma of the pancreas. Discussion of a rare epithelioid neoplasm. Pancreas. 2008;37:230-231.

4. Bancu VE, Vincze L, Kesztenbaum E, et al. Angioplastic sarcoma of the body of the pancreas; subtotal splenopancreatectomy. Chirurgia (Bucur). 1971;20:927-930.

5. Maeyashiki Ch, Nagata N, Uemura N. Angiosarcoma involving solid organs and the gastrointestinal tract with life-threatening bleeding. Case Rep Gastroenterol. 2012;6:772-2.

6. Meeks M, Grace S, Veerapong J, et al. Primary angiosarcoma of the pancreas. J Gastrointest Cancer. 2016 [Epub ahead of print]; DOI: 10.1007/ s12029-016-9837-1.

7. Young RJ, Brown NJ, Reed MW, et al. Angiosarcoma. Lancet Oncol. 2010;11:983-991.

8. Rossi S, Fletcher CD. Angiosarcoma arising in hemangioma/vascular malformation: report of four cases and review of the literature. Am J Surg Pathol. 2002;10:1319-1329.

9. Fury MG, Antonescu C, Van Zee KJ, et al. A 14-year retrospective review of angiosarcoma: clinical characteristics, prognostic factors, and treatment outcomes with surgery and chemotherapy. Cancer J. 2005;3:241-247.

10. Torres KE, Ravi V, Kin K, et al. Long-term outcomes in patients with radiation-associated angiosarcomas of the breast following surgery and radiotherapy for breast cancer. Ann Surg Oncol. 2013;20:1267-1274.

11. Meis-Kindblom J, Kindblom L. Angiosarcoma of soft tissue: a study of 80 cases. Am J Surg Pathol. 1995;22:683-697.

Cite this article as: Worth PJ, Turner M, Hammill CW (2017) Incidenta angiosarcoma of the pancreas: a case report of a rare, asymptomatic tumor, Journal of Pancreatic Cancer 3:1, 24-27, DOI: 10.1089/ pancan.2017.0007.

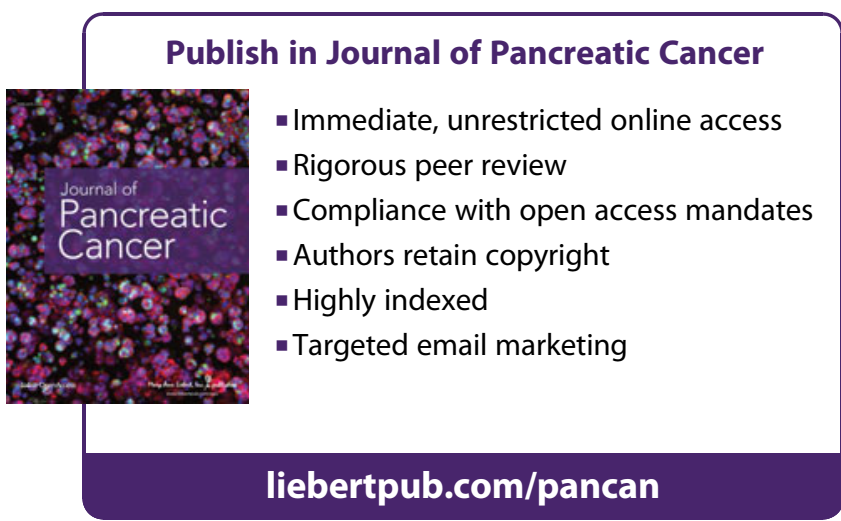

\section{Abbreviation Used}

$\mathrm{CT}=$ computed tomography 